remain rarely even though the initial attack manifests typical SAH in severity.

5. Prognosis is used to be benign and mortality rate is only $2.6 \%$ whereas $25.3 \%$ in aneurysm and $10 \%$ in $\mathrm{A}-\mathrm{V}$ malformation. All cases of $\mathrm{SAH}$ of unidentified etiology in this series survive and return to the previous profession except one.

Other multiple estimated etiologies were proposed by Sahs (1966) however there is no analogous case in our series except for one thrombocytopenic purpura as basic desease. The most possible cause of the unidentified SAH is thought to be the rupture of hidden micro-aneurysm on angiogram exceeding the level of usual angiographical analysis as pointed out by Krayenbuihl or due to the spontaneous thrombus formation in the ruptured aneurysm. As for the latter, we experienced $4 \times 4 \times 3 \mathrm{~mm}$ berry type aneurysm disappeared completely in the course of hospitalization which was clearly demonstrated on repeated angiography.

In addition, we succeeded in verifying the ruptured micropial vessel causing thin hemorrhage in the subarachnoidal space in autopsy terminated by the rupture of the another coexisting berry type aneurysm. This also indicates the possibility of such instance in case of the aged group with hypertensive atherosclerotic vascular changes.

\title{
h-9. Subarachnoid Hemorrhage due to rare causes
}

\author{
Junichi Kawafuchi, Tadashi Aiba, Fumikazu Takeda, Ichiro Handa, \\ Hisao YAGI and Norio Kono \\ Dept. of Neurosurgery, Gumma University
}

\section{Discussion to h-9.}

\section{Subarachnoid Hemorrhage Caused by Pituitary Adenoma}

\author{
Hideo Hiratsuka, Akira Kamisasa and Yutaka Inaba \\ Department of Neurosurgery, School of Medicine, Tokyo Medical and Dental University
}

Two cases of subarachnoid hemorrhage consequent to pituitary adenoma are reported. Case 1. A 32 year old female with history of amenorrhea, occasional headache and left visual disturbance was admitted because of sudden onset of severe headache, nausea, vomiting and amblyopia. On admission she was drowsy or stuporous. Neurological examination revealed right sided anosmia, amblyopia, papilledema, no reaction of pupils to light and left abducens paralysis. The neck was stiff to forward flexion. The CSF pressure was raised $(580 \mathrm{mmH} 20)$ 